Aim of the study: Post-radiogenic osteonecrosis of the mandible is a serious complication in patients with combined treated head/neck tumors. Osteonecrosis of the mandible can also occur following administration of bisphosphonates. In the present article we would like to present our experiences over the last five years in treating both bisphosphonate-associated osteonecrosis and osteoradionecrosis

Material and methods: Of the patients treated in our hospital for bone necrosis of the mandible between January 2005 and June 2010, 16 were diagnosed with infected osteoradionecrosis and 10 with bisphosphonate-associated osteonecrosis. The patients with osteoradionecrosis were administered the classic osteomyelitis treatment. The same procedure was carried out on 5 of the 10 patients with bisphosphonate-associated osteonecrosis; the remaining 5 were treated conservatively due to advanced bone infection. The bone biopsies and specimens of both entities yielded by the surgical interventions were examined histologically after decalcification.

Results: Of the 16 patients treated for infected osteoradionecrosis, 7 recovered after decortication and long-term antibiosis. In 7 cases consolidation did not occur until after osseous continuity resection. In 2 cases the progress of the intraosseous infection could not be stopped with treatment.

A typical first symptom of bisphosphonate-associated osteonecrosis was an alveolus that would not heal after a tooth extraction. In $50 \%$ of patients with bisphosphonate-associated osteonecrosis recovery was successful with a combination of surgery and long-term antibiosis. In the other patients with advanced bisphosphonate-associated osteonecrosis no definitive cure for the infection of the necrotic bone was possible.

Conclusion: In terms of treatment, osteoradionecrosis proves complex, yet easier to treat than bisphosphonate-associated osteonecrosis. The removal of the infected bone tissue is often necessary, but it does not always lead to recovery. Therefore it can be concluded that prevention of intraosseous infection by consistent pretherapeutic dental hygiene is especially important.

Key words: osteonecrosis, bisphosphonate, radiation, treatment, sequesterotomy.

\title{
Etiology and treatment of osteonecrosis of the mandible
}

\section{Florian Bast ${ }^{1}$, Andreas Groß², Lars Hecht ${ }^{3}$, Thomas Schrom²}

1Department of Oto-Rhino-Laryngology, University Hospital Charité Berlin, Campus Virchow-Klinikum, Berlin, Germany

${ }^{2}$ Clinic for Oto-Rhino-Laryngology, Head and Neck Surgery/Plastic Surgery, Helios Clinics Bad Saarow, Bad Saarow, Germany

3Institute for Pathology, Helios Clinics Bad Saarow, Bad Saarow, Germany

\section{Introduction}

Osteonecrosis of the mandible constitutes a serious complication following radiation treatment of head/neck tumors [1], chemotherapy for malignant diseases and treatment with bisphosphonates [2, 3]. Independent of its cause, osteonecrosis is defined as non-vital bone tissue resulting from disrupted intraosseous blood supply. This leads to trophic damage of the bone and the demise of osteocytes and at the same time to the consecutive degeneration and destruction of the bone matrix.

Radiation of malignancies in the head and neck region, either alone or in combination with surgery and/or chemotherapy, is in many cases an essential component of cancer treatment. Depending on the location of the radiation field and the dose, more or less progredient and ultimately irreversible damage to the bones and vessels occurs. Because of the loss of immune function, radiation-damaged bone is vulnerable to germs found in the oral cavity and therefore to infection. When a secondary infection arises after odontogenic or traumatic procedures, the result, due to disrupted immune function and reduced vitality, can be infected osteonecrosis. In the literature the incidence of this development, depending on the radiation dose and form, is estimated at between 1 and 20 percent, and the occurrence of osteoradionecrosis is described from one month to 14 years post-radiation [4-8]. Risk factors for the development of osteoradionecrosis are above all the tumor stage and the necessary radiation dose, the location of the radiation field, age, tooth condition and necessary tooth extraction shortly before or after radiation treatment. According to the literature the average age of patients with osteoradionecrosis is over 55 years [4, 8-10]. Curi et al. noted that the causes of the development of infected osteoradionecrosis, at over 60\%, are "tooth, mouth or jaw-related" [11]

Even in the 19th century, painful necroses of the mandible ("phossy jaw") were described in factory workers involved in the production of matches containing phosphorous. As a result the Berne Convention (1906) ended the production and sale of phosphorous matches in most countries in the world [12]. Bisphosphonates - chemical compounds made up of two phosphonate groupshave been used for decades in the standard treatment of bone metabolism dysfunction, for example bone metastasis arising as part of a malignant primary disease, osteoporosis, or Morbus Paget [13]. Bisphosphonates are normally administered intravenously for bone metastasis treatment, and orally for osteoporosis treatment [3]. In the treatment of Morbus Paget bisphosphonates are both orally and intravenously administered. The main effect of bisphosphonates is the inhibition of osteoclastic activity and the resulting reduction of bone resorption. Marx in 2003 first described osteonecrosis of the lower jaw as a serious side effect of bisphosphonate treatment [14]. At the time the incidence was estimated at $3-10 \%$, and growing. The cumulative effect is espe- 
cially problematic because of the long half-life of bisphosphonates from months to years [15-18]. In bisphosphonateassociated osteonecrosis, the mandible is affected, with exposed bone not prone to spontaneous healing. Multi-factor development is evident. The first important factor is the patient's primary disease, whereby patients with multiple myelomas run the highest risk [19]. The potency of the bisphosphonates, how they are administered and the length of treatment also influence the development of osteonecrosis [20]. Another factor is dental history, including interventions, treatments and hygiene $[17,19,21]$. Osteonecrosis arising from these factors is based on the walls of the Haversian canals. As a result of stronger resistance to bone depletion as compared to bone formation, a positive bone balance may result; however, if blood flow to the bone is reduced, the demise of osteocytes will ensue. According to the literature the average age of patients with bisphosphonate-associated osteonecrosis is over 60 years [4, 22].

Infected osteonecrosis is the most serious form of osteomyelitis. Characteristic of this condition is the occurrence of extensive exposed bone areas, in part associated with intra- and extraoral fistulas, extreme pain and comprehensive osteolysis and even pathological fractures [4, 20].

Treatment of osteonecrosis, independent of the etiology, is a subject of debate. In general, the most important measure is considered to be prophylaxis prior to systemic bisphosphonate administration or radiation. Chronic infectious processes in the intraoral mucous membrane and jaw area should be corrected before treatment. In addition, close observation during treatment is indicated.

When osteonecrosis is evident, antibiotic treatment, possibly in combination with surgical procedures, should always be carried out. The goal here is to remove non-vital bone, cover exposed bone, and preserve unaffected and adjacent bones and organs in order to prevent a relapse [10, 17, 23]. Hyperbaric oxygen therapy is hotly debated in the literature [24-26].

In the present article we would like to present our experiences over the last five years in treating both bisphosphonate-associated osteonecrosis and osteoradionecrosis.

\section{Material and methods}

All 26 patients treated in our hospital for osteonecrosis of the mandible between January 2005 and June 2010 were included in the study. The patient group was composed of 4 women and 22 men ranging in age from 42 to 76 (average age: 60.7$)$.

Patients were grouped according to the etiology of the osteonecrosis. 16 patients (15 men and 1 woman) were suffering from infected osteoradionecrosis, and 10 patients (7 men and 3 women) had infected bisphosphonate-associated osteonecrosis. 8 patients with an osteoradionecrosis and 6 patients with a bisphosphonate-associated osteonecrosis had a tooth extraction lately.

In addition to a thorough clinical examination, blood tests and an orthopantomogram to determine the size and degree of the osteonecrosis (Fig. 1) were carried out. In order to ensure correct diagnosis a bone biopsy and histological evaluation were also performed.

Patients were treated with the classic therapy steps for osteomyelitis. The step-by-step treatment includes procedures based on medical findings, which comprise care and cleansing of the area, systemic antibiosis and surgical resection.

In our patient population the first step was always longterm antibiosis as a sequence therapy. The preferred antibiotic was clindamycin (3x daily 600 mg i.v.) due to its suitability for bone and its efficacy spectrum. In cases of intolerance or extreme, mainly gastrointestinal complaints, cefuroxime ( $3 \times$ daily $1.5 \mathrm{~g}$ i.v.) was substituted. The medication was administered intravenously on an in-patient basis and was changed to oral form at discharge. When recovery after a minimum of 4 weeks of antibiosis was not achieved, a surgical procedure was carried out involving intubation and anesthesia and consisting of decortication with sequesterotomy and modulated osteotomy, or even partial or total resection of the affected mandible.

\section{Results}

All patients first received antibiotic therapy independent of the etiology of their condition. 20 patients were admin-

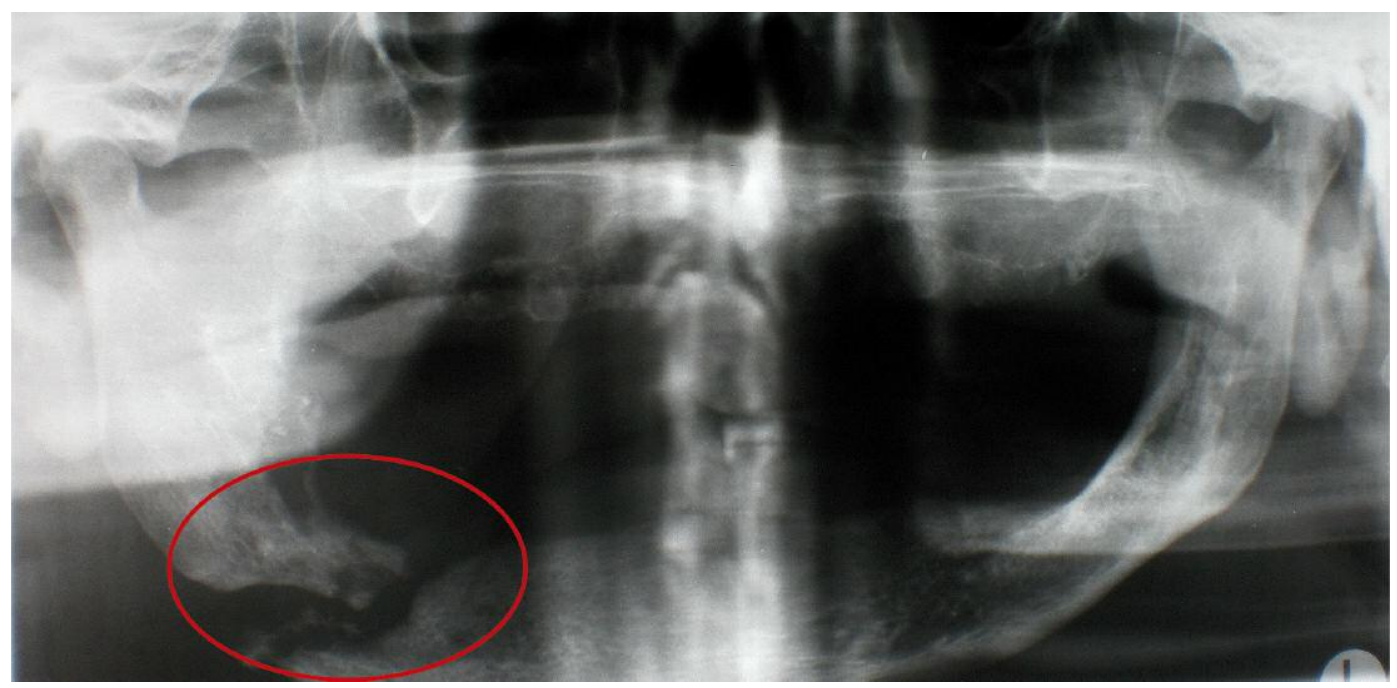

Fig. 1. Orthopantomogram with pathologic corpus mandibulae fracture in right-sided osteoradionecrosis 
istered clindamycin intravenously $3 \times 600 \mathrm{mg}$. The other 6 patients received cefuroxime intravenously $3 \times 1.5 \mathrm{~g}$ due to intolerance of clindamycin.

Of the patients with infected osteoradionecrosis 7 recovered after decortication and long-term antibiosis (7 weeks). The other 9 patients experienced a relapse despite successful treatment, and twice pathologic lower jaw fractures (Fig. 1). As a result of the relapses, clindamycin or cefuroxime was again administered and a surgical continuity resection was performed on the lower jaw. With the combined treatment, 7 patients recovered; in the other 2 the intraosseous infection continued to progress despite treatment. In these cases a long-term local treatment was carried out for acute exacerbation of the infection. Thus over $80 \%$ of patients with osteoradionecrosis recovered after intensive treatment.

The typical first symptom of bisphosphonate-associated osteonecrosis in over $60 \%$ of the patients was an alveolus, which would not heal after tooth extraction. In $50 \%$ of patients with bisphosphonate-associated osteonecrosis, recovery was successful after a combination of long-term antibiosis with clindamycin or cefuroxime and surgery involving decortication. The other 5 patients were only given local treatment due to the advanced stage of the bone infection. In case of exacerbation of the infection, antibiosis with clindamycin or cefuroxime was administered, in addition to antiseptic rinsing and isolated removal of bone sequesters. These patients did not experience a full recovery and in 3 cases pathological fractures occurred.

The bone biopsies and samples of both entities taken during the surgical procedures were histologically analyzed after decalcification. Two distinct and reproducible reaction patterns of the tissue were identified, according to previous treatment. The high cytotoxicity of the radiation led directly to osteonecrosis, which after resorption was replaced with scar tissue (Fig. 2).

Bisphosphonates first induced increased osteogenesis leading to the walling in of the Haversian canals and the accompanying reduction of the total vessel width. Osteonecrosis developed in areas with especially poor nutritive conditions (Fig. 3a). In addition, the presence of Actinomyces drusen

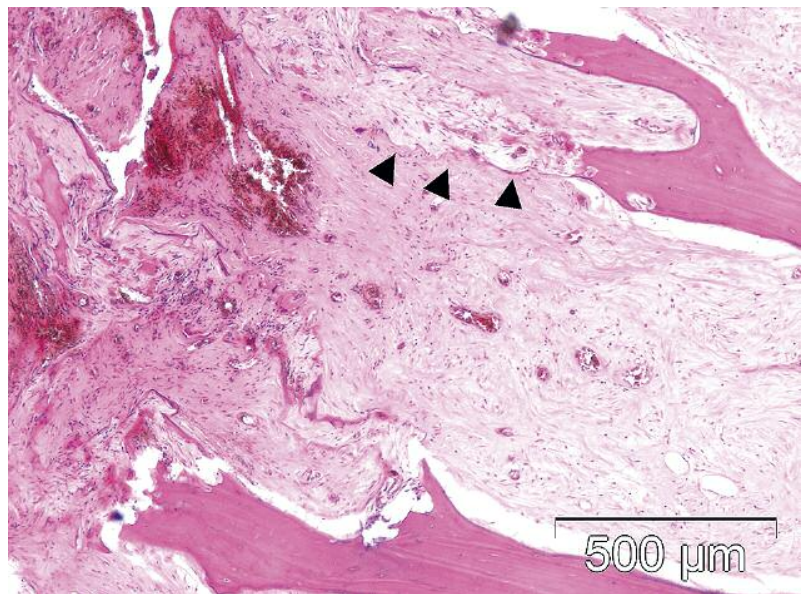

Fig. 2. Spongy bone with radiogenic necrosis in resorption. Recognizable trabecular contour $(\boldsymbol{A})$ changing over to scar tissue (HEdye, EDTA-decalcification)

was characteristic of bisphosphonate-associated osteonecrosis (Fig. 3b) [27].

\section{Discussion}

Osteonecrosis occurs as a result of a disrupted intraosseous blood supply and involves trophic damage to the bone with the demise of osteocytes to non-vital bone tissue and bacterial proliferation. It is mainly a consequence of radiation treatment of head and neck tumors or of systemic bisphosphonate treatment. Osteonecrosis of the jaw due to radiation or treatment with bisphosphonates occurs only in adults [4].

Osteonecrosis following radiation of head and neck tumors represents a well-known and often described complication of tumor treatment [10]. Depending on the location of the radiation field and dose, the bone is subject to more or less progredient and ultimately irreversible damage. The threshold dose is given as 60 Gy in the literature. Patients with $\mathrm{T} 4$ tumors that require higher doses of radiation are espe-
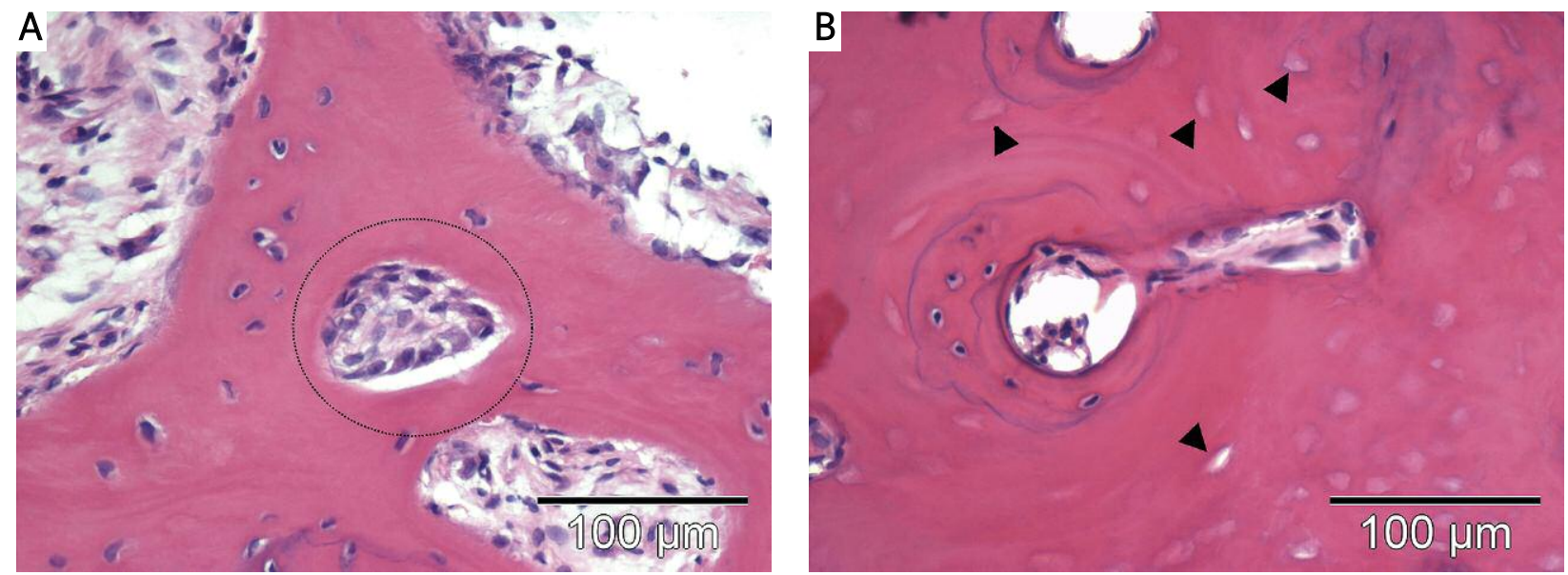

Fig. 3. A) Haversian canal almost completely filled with osteoblasts (HE-dye, EDTA-decalcification); B) Haversian canal with hypoplastic blood vessel and shell-like vital bone area. Non-vital bone with adjacent empty osteocyte cavities ( $\mathbf{\Delta}$ ) (HE-dye, EDTA-decalcification) 

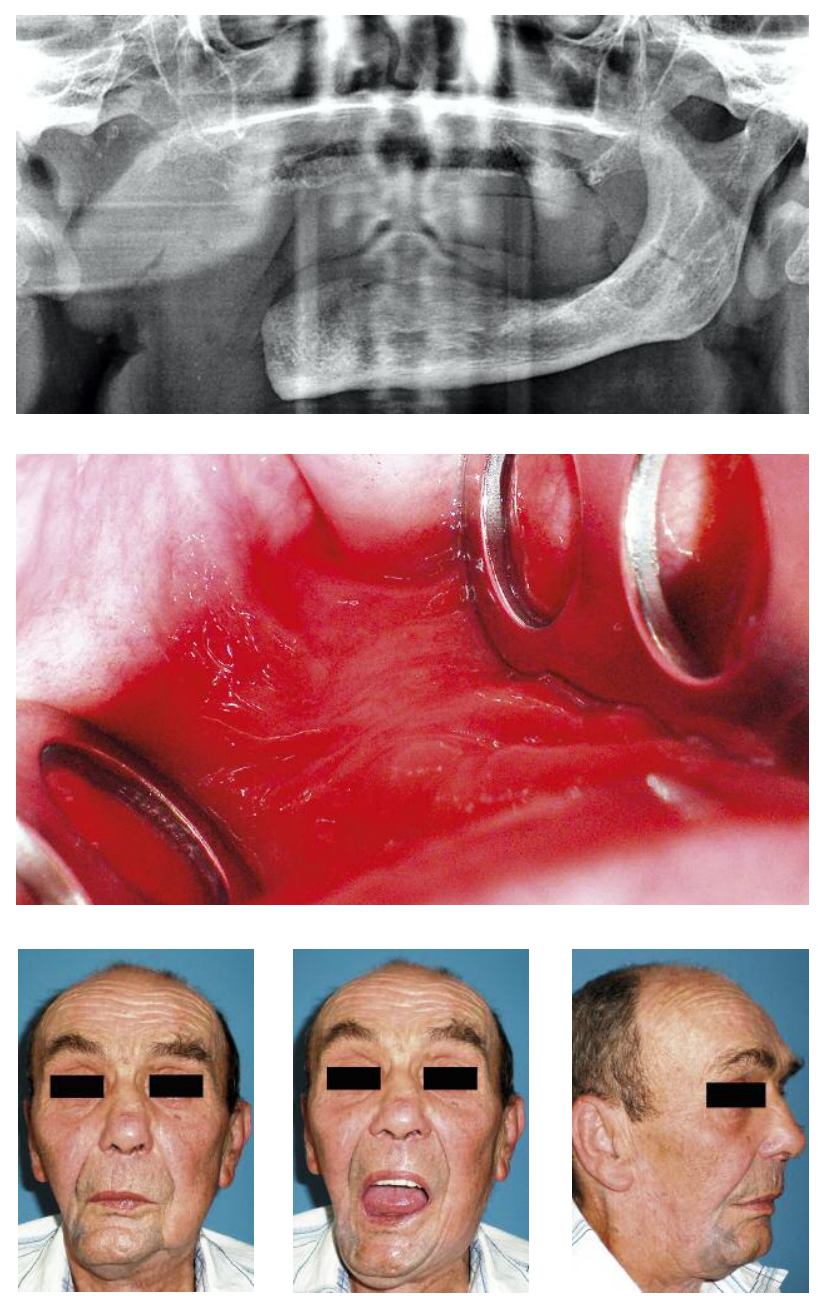

Fig. 4. Orthopantomogram, intraoral findings and functionality after treatment of osteoradionecrosis of the right lower jaw

cially susceptible to osteonecrosis, which can occur anywhere from one month to 14 years after radiation therapy [4].

Bisphosphonates have been used for decades in the standard treatment of bone metabolism dysfunction or bone metastasis deriving from a primary malignancy, of osteoporosis or of Morbus Paget [13]. In 2003 case studies of atypical necrosis of the mandible first appeared. Lesions normally occur as intraoral exposed bone wherein the lower jaw is more often affected than the upper [14].

The development of osteonecrosis is promoted by other factors in addition to radiation or bisphosphonate exposure. Among these are dentogenic infections, soft tissue and bone wounds especially after tooth extraction or poorly situated prostheses as well as immunosuppressive treatments. As a result, prevention measures prior to radiation or bisphosphonate treatment are particularly important. Before such treatment begins, thorough odontological care and cleaning of the teeth and mucous membrane should take place and a radiation protector or fluoridation splint should be fitted. An examination of the oral cavity should also ensure that no pressure points from removable dentures are present [28]. These measures, according to Grötz et al. can reduce the likelihood of osteoradionecrosis by a factor of 3 [29].
If however, during or after radiation or bisphosphonate treatment, an infected osteonecrosis occurs, treatment is urgently needed. The classic pillars of osteomyelitis treatment are conservative long-term antibiosis as a sequence treatment, followed by surgical interventions and possibly hyperbaric oxygen therapy ( $\mathrm{HBO}$ ), although the latter is hotly debated in the literature. Current reviews and studies do not assume a positive outcome for HBO therapy [24-26].

All in all, osteonecrosis, whatever the etiology, is a relatively rare but serious complication. Our patient population showed that osteoradionecrosis was complex, but still easier to treat than bisphosphonate-associated osteonecrosis.

Following long-term antibiosis and in some cases surgical procedures, more than $80 \%$ of the patients suffering from osteoradionecrosis fully recovered. 9 patients underwent a continuous resection of the affected lower jaw. Because functionality in our patients returned to a high level after recovery, no further reconstruction measures were performed (Fig. 4). The shortest post-study period is 7 months.

With the same procedures we were only able to achieve full recovery in $50 \%$ of the patients from our population with bisphosphonate-associated osteonecrosis. The shortest post-study period for this group is 8 months.

These figures confirm data found in the literature $[4,11$, $27,30]$. We attribute the difficult nature of the treatment for bisphosphonate-associated osteonecrosis to the systemic character of the illness, since the bone is not locally but rather systemically affected and is generally disrupted in its osteoblast and osteoclast activity.

The histological analysis of the bone samples showed that patients with bisphosphonate-associated osteonecrosis displayed Actinomyces in the bone, which is also described in the literature [27]. In addition, however, due to a positive bone balance, strongly walled Haversian canals were found. The reduced vessel width resulting from this leads to reduced blood and oxygen supply to the bone and in turn to the demise of osteocytes and poor immune function. Not until this occurs is the presence of Actinomyces possible. Regarding histological changes caused by treatment with bisphosphonates, the literature includes varying opinions [27, 31, 32].

For those patients who did not experience a full recovery after combined treatments, we do not intend to carry out $\mathrm{HBO}$ because of its uncertain medical status and the fact that insurance companies do not cover its costs [24-26].

Due to the complex and difficult treatment for infected osteonecrosis, especially when associated with bisphosphonates, prophylactic measures are highly valued. Thus intense, interdisciplinary cooperation prior to the start of radiation or bisphosphonate therapy is essential for patients and for economic reasons.

In conclusion, in terms of treatment, osteoradionecrosis is complex, yet easier to treat than bisphosphonate-associated osteonecrosis. The removal of infected bone tissue is often necessary, but does not always lead to a full recovery. For this reason prevention of intraosseous infections by means of consistent pre-treatment dental hygiene is of special importance.

The authors declare no conflict of interest. 


\section{References}

1. van Kaick G, Delorme S. Therapy-induced effects in normal tissue. Radiologe 2008; 48: 871-80.

2. Marx RE, Cillo JE, Jr, Ulloa JJ. Oral bisphosphonate-induced osteonecrosis: risk factors, prediction of risk using serum CTX testing, prevention, and treatment. J Oral Maxillofac Surg 2007; 65: 2397-410.

3. Pazianas M, Miller P, Blumentals WA, Bernal M, Kothawala P. A review of the literature on osteonecrosis of the jaw in patients with osteoporosis treated with oral bisphosphonates: prevalence, risk factors, and clinical characteristics. Clin Ther 2007; 29: 1548-58.

4. Almazrooa SA, Woo SB. Bisphosphonate and nonbisphosphonate associated osteonecrosis of the jaw: a review. J Am Dent Assoc 2009; 140: 864-75.

5. Studer G, Gratz KW, Glanzmann C. Osteoradionecrosis of the mandibula in patients treated with different fractionations. Strahlenther Onkol 2004; 180: 233-40.

6. Studer G, Studer SP, Zwahlen RA, Huguenin P, Grätz KW, Lütolf UM, Glanzmann C. Osteoradionecrosis of the mandible: minimized risk profile following intensity-modulated radiation therapy (IMRT). Strahlenther.Onkol 2006; 182: 283-8.

7. Sciubba JJ, Goldenberg D. Oral complications of radiotherapy. Lancet Oncol 2006; 7: 175-83.

8. Reuther T, Schuster T, Mende U, Kübler A. Osteoradionecrosis of the jaws as a side effect of radiotherapy of head and neck tumour patients - a report of a thirty year retrospective review. Int J Oral Maxillofac Surg 2003; 32: 289-95.

9. Grotz KA, Riesenbeck D, Brahm R, et al. Chronic radiation effects on dental hard tissue (radiation caries). Classification and therapeutic strategies. Strahlenther Onkol 2001; 177: 96-104.

10. Pitak-Arnnop P, Sader R, Dhanuthai K, Masaratana P, Bertolus C, Chaine A, Bertrand JC, Hemprich A. Management of osteoradionecrosis of the jaws: an analysis of evidence. Eur J Surg Oncol 2008; 34: 1123-34.

11. Curi MM, Dib LL Osteoradionecrosis of the jaws: a retrospective study of the background factors and treatment in 104 cases. J Oral Maxillofac Surg 1997; 55: 540-4.

12. Emsley J. Phosphor - ein Element auf Leben und Tod. Wiley-VCH, Weinheim 2001; 63-122.

13. Aapro MS. Management of bisphosphonate treatment in clinical practice. Semin Oncol 2007; 34 (6 Suppl 4): S28-S32.

14. Marx RE. Pamidronate (Aredia) and zoledronate (Zometa) induced avascular necrosis of the jaws: a growing epidemic. J Oral Maxillofac Surg 2003; 61: 1115-7.

15. Bamias A, Kastritis E, Bamia C, et al. Osteonecrosis of the jaw in cancer after treatment with bisphosphonates: incidence and risk factors. J Clin Oncol 2005; 23: 8580-7.

16. Durie BG, Katz M, Crowley J. Osteonecrosis of the jaw and bisphosphonates. N Engl J Med 2005; 353: 99-102.

17. Ruggiero SL, Dodson TB, Assael LA, Landesberg R, Marx RE, Mehrotra B; American Association of Oral and Maxillofacial Surgeons. American Association of Oral and Maxillofacial Surgeons position paper on bisphosphonate-related osteonecrosis of the jaws--2009 update. J Oral Maxillofac Surg 2009; 67 (5 Suppl): 2-12.

18. Mavrokokki T, Cheng A, Stein B, Goss A. Nature and frequency of bisphosphonate-associated osteonecrosis of the jaws in Australia. J Oral Maxillofac Surg 2007; 65: 415-23.

19. Marx RE, Sawatari Y, Fortin M, Broumand V. Bisphosphonateinduced exposed bone (osteonecrosis/osteopetrosis) of the jaws: risk factors, recognition, prevention, and treatment. J.Oral Maxillofac Surg 2005; 63: 1567-75.

20. Woo SB, Hellstein JW, Kalmar JR. Narrative [corrected] review: bisphosphonates and osteonecrosis of the jaws. Ann Intern Med 2006; 144: 753-61.

21. Dannemann C, Gratz KW, Riener MO, Zwahlen RA. Jaw osteonecrosis related to bisphosphonate therapy: a severe secondary disorder. Bone 2007; 40: 828-34.

22. Grötz KA, al-Nawas B, Brahm R, Duschner H, Wagner W. Terminal end of the human odontoblast processes. Clin.Oral Investig 2000; 4: 106-7.

23. Rhodus NL. Management of oral complications from radiation and chemotherapy. Northwest.Dent. 2010; 89: 39-42.
24. Pitak-Arnnop P, Hemprich A, Dhanuthai K, Pausch NC. A systematic review in 2008 did not show value of hyperbaric oxygen therapy for osteoradionecrosis. J Oral Maxillofac Surg 2010; 68: 2644-5.

25. Fritz GW, Gunsolley JC, Abubaker O, Laskin DM. Efficacy of pre- and postirradiation hyperbaric oxygen therapy in the prevention of postextraction osteoradionecrosis: a systematic review. J Oral Maxillofac Surg 2010; 68: 2653-60.

26. Bessereau J, Annane D. Treatment of osteoradionecrosis of the jaw: the case against the use of hyperbaric oxygen. J Oral Maxillo

27. Abu-Id MH, Açil Y, Gottschalk J, Kreusch T. Bisphosphonate-associated osteonecrosis of the jaw. Mund Kiefer Gesichtschir 2006; 10: 73-81.

28. Hellstein JW, Marek CL. Bisphosphonate induced osteochemonecrosis of the jaws: an ounce of prevention may be worth a pound of cure. Spec Care Dentist 2006; 26: 8-12.

29. Grötz KA, Wüstenberg P, Kohnen R, et al. Prophylaxis of radiogenic sialadenitis and mucositis by coumarin/troxerutine in patients with head and neck cancer - a prospective,randomized, placebo-controlled, double-blind study. Br J Oral Maxillofac Surg 2001; 39: 34-9.

30. Bagan JV, Jiménez Y, Hernández S, et al. Osteonecrosis of the jaws by intravenous bisphosphonates and osteoradionecrosis: a comparative study. Med Oral Patol Oral Cir Bucal 2009;14: e616-9.

31. Hellstein JW, Marek CL. Bisphosphonate osteochemonecrosis (bisphossy jaw): is this phossy jaw of the 21st century? J Oral Maxillofac Surg 2005; 63: 682-9.

32. Hellstein JW, Marek CL. Bis-phossy jaw, phossy jaw, and the 21st century: bisphosphonate-associated complications of the jaws. J Oral Maxillofac Surg 2004; 62: 1563-5.

\section{Address for correspondence}

\section{Dr. med. Florian Bast}

University Hospital Charité Berlin

Department of Oto-Rhino-Laryngology

Campus Virchow-Klinikum

Augustenburger Platz 1

13353 Berlin, Germany

tel. +49 (0) $30450-655026$

$\mathrm{fax}+49$ (0) 30 450-555900

e-Mail: Florian.Bast@charite.de

Submitted: $\quad 17.09 .2012$

Accepted: $\quad 12.02 .2013$ 